\title{
Output Capacitance Losses in Wide-Band-Gap Transistors: A Small-Signal Modeling Approach
}

\author{
Mohammad Samizadeh Nikoo*, Armin Jafari, Nirmana Perera, and Elison Matioli* \\ Power and Wide-band-gap Electronics Research Laboratory (POWERlab) \\ École Polytechnique Fédérale de Lausanne (EPFL) \\ Lausanne, Switzerland \\ *mohammad.samizadeh@epfl.ch, elison.matioli@epfl.ch
}

\begin{abstract}
Wide-band-gap (WBG) power semiconductor devices are gaining an increasing interest in power circuits, as they exhibit a low specific ON-resistance (RON) while providing a high blocking voltage. The energy dissipation corresponding to resonantly charging and discharging their output capacitance (Coss), however, severely limits their performance at high switching frequencies. In this work, we demonstrate a simple approach based on a small-signal measurement, to model $C_{\text {oss }}$ losses in transistors. The device output capacitance is modeled by an effective $C_{0 s s}$ in series with a frequency-dependent resistance $R_{\mathrm{s}}$. The proposed method is completely based on a small-signal measurement and it directly leads to a general view of frequencydependent $C$ oss losses in power transistors. We consider four commercial devices based on $\mathrm{GaN}$ and $\mathrm{SiC}$, and using the proposed technique, we evaluate $C_{\text {oss }}$ losses. We verify the model-based prediction with thermal measurements. The precise characterization of $C$ oss-losses proposed in this paper is essential for designing efficient high-frequency power converters.
\end{abstract}

Keywords-Wide-band-gap, GaN, SiC, high frequency, output capacitance, Coss losses.

\section{INTRODUCTION}

Using wide-band-gap (WBG) semiconductor devices leads to considerable reduction of conduction losses in power converters with respect to Si devices, as they provide a lower specific ON-resistance [1]. WBG semiconductor devices also exhibit a significantly lower gate capacitance with respect to their Si counterparts, which enables WBG devices to operate at much higher switching frequencies. This leads to a considerable size reduction in passive components, therefore, a much higher power density can be achieved [2]. This property together with the ease of integration, also paves the way towards monolithically integrated power circuits, which operate at megahertz switching frequencies [3].

The recently observed energy dissipation caused by resonantly charging and discharging of the output capacitance ( $\left.C_{\text {OSS }}\right)$ of WBG semiconductor devices, however, severely limits their performance at high switching frequencies [4]-[8]. This can result in significantly lower-than-expected efficiencies in power converters [9]. Using large-signal measurement methods such as Sawyer-Tower (ST) [4] and nonlinear resonance [7], a frequency-dependent energy dissipation was measured in WBG transistors. This was in contrary to the previously observed frequency-independent $C_{\text {OSs }}$ losses in $\mathrm{Si}$ superjunction (SJ) transistors [10], [11].

In all of the commonly used large-signal measurement techniques of $C_{\text {oss }}$ losses, separate measurements are needed at different operation points, including voltage-swing, frequency, and $\mathrm{d} v / \mathrm{d} t$, which makes it difficult to obtain a full view of $C_{\text {oss }}$ losses in different devices. Furthermore, practical constraints such as power-frequency trade-off in power amplifiers, can substantially limit the loss characterization in transistors especially at high frequencies. A recently proposed method based on a small signal modeling approach, suggested a simple way to fully characterize the frequency-dependent $C_{\text {oss }}$ losses in WBG transistors [8]. The device output capacitance can be modeled by an effective $C_{\mathrm{OSS}}\left(C_{\mathrm{OSS}}^{\mathrm{eff}}\right)$ in series with a resistance $R_{\mathrm{S}}$. These parameters, which can be easily measured by an impedance analyzer, show a complete view of frequencydependent $C_{\text {OSS }}$ losses in power transistors. In this work we demonstrate the selection of devices with lowest $C_{\text {oss }}$ losses based on this modeling approach, just using a simple smallsignal measurement, and corroborate them with thermal measurements.

\section{SMALL-SIGNAL MODEL}

Fig. 1a shows the proposed model for output capacitance, including a nonlinear capacitance in series with resistance $R_{\mathrm{S}}$ and in parallel with resistance $R_{\mathrm{P}}$. The effect of $R_{\mathrm{P}}$ is dominant at $\mathrm{DC}$, while $R_{\mathrm{S}}$ significantly contributes to high-frequency

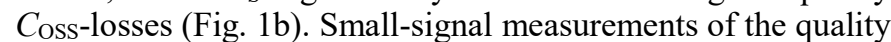
factor ( $Q$-factor) of the $C_{\text {oss }}$ show a considerable lossy behavior for $C_{\mathrm{OSS}}$ of WBG transistors (Fig. 1c). By applying voltage $v(t)$ to the output capacitance, and considering $R_{\mathrm{S}}$ as a perturbation element, the power loss in $R_{\mathrm{S}}$ can be written as $P_{\text {DISS }}=R_{\mathrm{S}}\left(C_{\mathrm{OSS}} \mathrm{d} v / \mathrm{d} t\right)^{2}$. Considering an operation frequency $f$, we obtain the charging/discharging $C_{\text {oss }}$ energy dissipation as

$$
E_{\mathrm{DISS}}=2 R_{\mathrm{S}}\left(\frac{\mathrm{d} v}{\mathrm{~d} t}\right) \int_{0}^{V} C_{\mathrm{OSS}}^{2} \mathrm{~d} v
$$

(C) 2020 IEEE. Personal use of this material is permitted. Permission from IEEE must be obtained for all other uses, in any current or future media, including reprinting/republishing this material for advertising or promotional purposes, creating new collective works, for resale or redistribution to servers or lists, or reuse of any copyrighted component of this work in other works. 
(a)

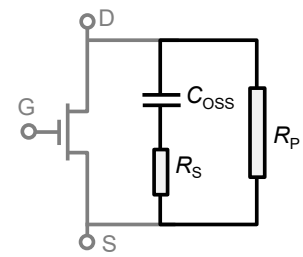

* The nonlinear $C_{\text {OSS }}\left(V_{D S}\right)$ can be replaced by a fixed capacitance $C_{\text {OSS }}^{\text {eff }}$.

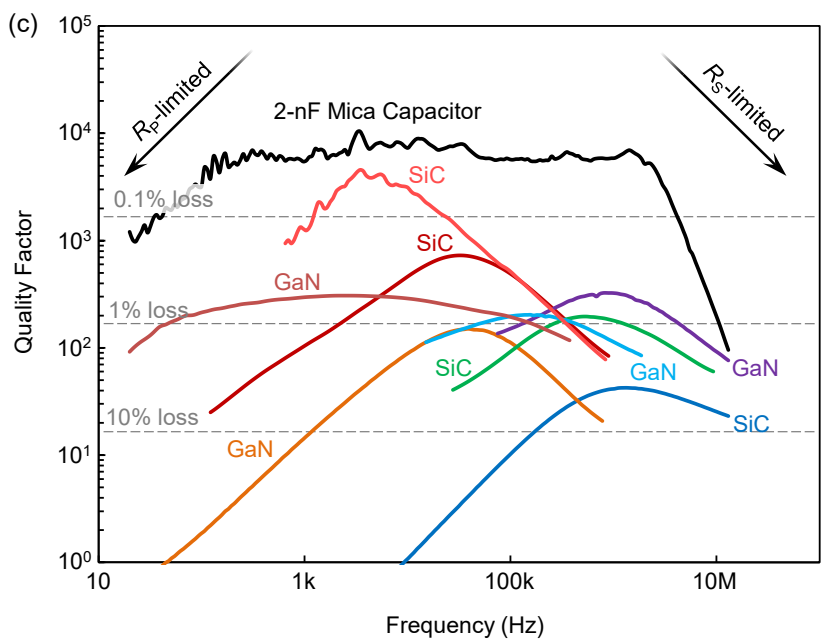

Fig. 1. (a) A model for output capacitance of transistors (OFF state) where $C_{\text {OSS }}$ is a nonlinear voltage-dependent capacitance and $R_{\mathrm{S}}$ and $R_{\mathrm{P}}$ represent losses at low and high frequencies, respectively. (b) Schematic of qualityfactor ( $Q$-factor) of output capacitance of a transistor representing the amount of $C_{\mathrm{OSS}}$ losses. The effect of $R_{\mathrm{P}}$ (mainly corresponding to the leakage current) is dominant at DC, while $R_{\mathrm{S}}$ significantly contributes to the switching dynamics and $C_{\text {Oss }}$ losses. (c) Measured $Q$-factor of output capacitance of several commercial WBG transistors versus frequency at $V_{\mathrm{DS}}=40 \mathrm{~V}$. The gate and source of transistors where shorted $\left(C_{\mathrm{OSS}}=C_{\mathrm{DS}}+C_{\mathrm{GD}}\right)$. The amount of losses $(2-10 \%)$ is in agreement with the previously measured losses using large-signal methods [4]-[7].

which shows a $\mathrm{d} v / \mathrm{d} t$-dependence of $E_{\text {DIss. }}$ Considering a sinusoidal excitation with frequency $f$ we have

$$
E_{\mathrm{DISS}}=4 R_{\mathrm{S}} f V^{2} C_{\mathrm{OSS}}^{\mathrm{eff} 2}
$$

where we introduce the following term of effective $C_{\text {OSS }}$, related to the portion of $C_{\text {OSS }}$ that contributes to power dissipation:

$$
C_{\mathrm{OSS}}^{\mathrm{eff}}=\sqrt{\frac{1}{V} \int_{0}^{V} C_{\mathrm{OSS}}^{2} \mathrm{~d} v}
$$

Equation (2) represents $E_{\text {DISS }}$ exclusively as a function of $R_{\mathrm{S}}$ (which can be measured) and $C_{\mathrm{OSS}}^{\text {eff }}$ (which can be extracted from datasheet).

Fig. 2 shows three steps for $E_{\text {DISS }}$ extraction based on the introduced method, for a 1200-V 36-A SiC MOSFET. In the (a) Step 1: Extraction of $C_{\text {OSS }}^{\text {eff }}$

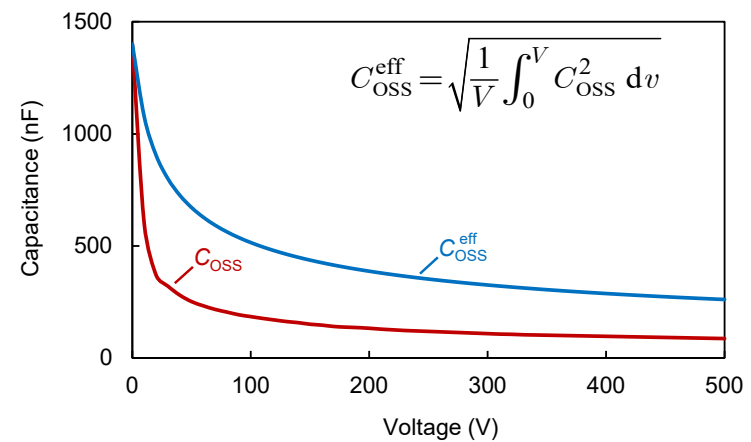

(b) Step 2: Measurement of $R_{\mathrm{S}}$

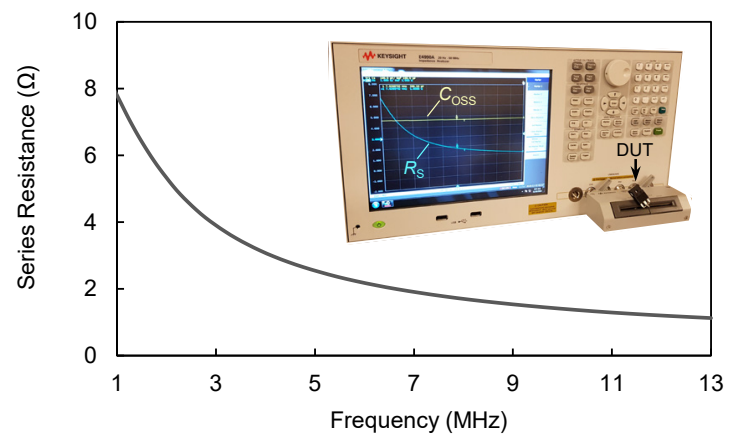

(c) Step 3: $C_{\text {oss }}$ loss calculation

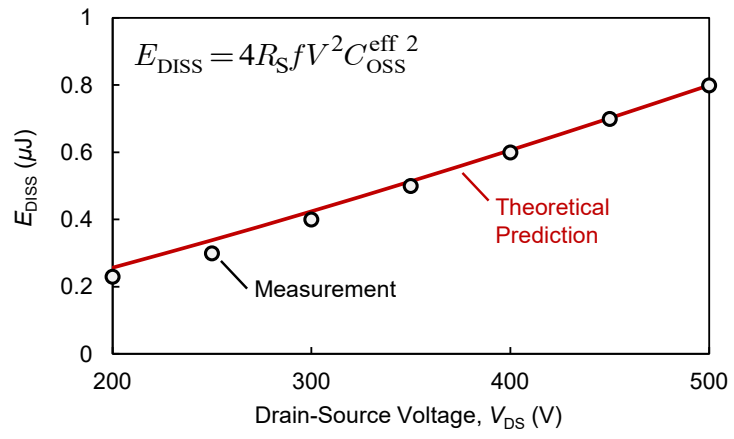

Fig. 2. Procedure of $C_{\text {Oss }}$ loss evaluation in wide-band-gap transistors. (a) In the first step, the effective $C_{\text {oss }}$ is extracted using (3) based on data reported in datasheet. (b) The series resistance $R_{\mathrm{S}}$ measured at $40 \mathrm{~V}$ versus frequency is measured in the next step. The inset shows the measurement set-up with a E4990A 50-MHz impedance analyzer. (c) Using (1), and based on the effective $C_{\mathrm{OSS}}$ and $R_{\mathrm{S}}$, the $C_{\mathrm{OSS}}$ energy dissipation can be calculated. The results corresponding to the frequency of $1-\mathrm{MHz}$ is illustrated (solid line) showing a good agreement with large-signal measurements based on ST method.

first and second steps, $C_{\mathrm{OSS}}^{\text {eff }}$ and $R_{\mathrm{S}}$ are obtained. The data presented in datasheet can be used to extract $C_{\text {OSS }}^{\text {eff }}$ (Fig. 2a), while $R_{\mathrm{S}}$ should be measured with an impedance analyzer (Fig. 2b). In the third step, $E_{\text {DISS }}$ is calculated using (1). To verify the predicted results, we compared the results corresponding to the frequency of $1-\mathrm{MHz}$, with large-signal measurement results, which shows an excellent agreement (Fig. 2c). 


\section{Selection of DeVices With Lowest C Coss Losses}

The small-signal method to evaluate $E_{\text {DISS }}$ is a simple technique showing a general view of $C_{\text {OSs }}$ losses in WBG transistors and, therefore, can be used to select low-loss devices. Among WBG transistors within the same $R_{\mathrm{ON}}$ and voltage rating, the values of $C_{\mathrm{OSS}}$ are generally the same; hence, the value of $R_{\mathrm{S}}$ determines the level of $C_{\text {OSS }}$ losses. As a result, a single measurement determines the device with lowest amount of $C_{\text {oss }}$ losses, which is preferable for high switching frequency power circuits.

We selected four WBG transistors with similar current capability of $\sim 30-\mathrm{A}$ based on $\mathrm{GaN}$ (devices $\mathrm{A}$ and $\mathrm{B}$ ) and $\mathrm{SiC}$ (devices $\mathrm{C}$ and D). A detailed description of the selected devices is presented in Table I. Figs. 3a-d represent the measured Coss versus voltage at two different frequencies 1$\mathrm{MHz}$ and 10-MHz, for devices A-D, respectively. These figures show that all of the selected devices have the same range of $C_{\text {OSs }}$ values, and the measurement results agree with the values reported in datasheet. Fig. 3e shows the measured $R_{\mathrm{S}}$
TABLE I

SPECIFICATIONS OF EVALUATED WBG TRANSISTORS

\begin{tabular}{|c|c|c|c|c|c|}
\hline \multirow{2}{*}{ Device } & \multirow{2}{*}{ Type } & \multicolumn{2}{|c|}{ "Voltage and current rating } & \multirow{2}{*}{$\begin{array}{l}R_{\mathrm{ON}}{ }^{* *} \\
(\mathrm{~m} \Omega)\end{array}$} & \multirow{2}{*}{$\begin{array}{l}C_{\mathrm{OSS}}(\mathrm{pF}) \\
\text { at } 400-\mathrm{V}\end{array}$} \\
\hline & & Voltage (V) & Current $^{*}(\mathrm{~A})$ & & \\
\hline $\mathrm{A}$ & $\mathrm{GaN}$ & \multirow{2}{*}{600} & 31 & 55 & 72 \\
\hline $\mathrm{B}$ & FET & & 26 & 56 & 71 \\
\hline $\mathrm{C}$ & $\mathrm{SiC}$ & \multirow{2}{*}{650} & 30 & 80 & 66 \\
\hline $\mathrm{D}$ & FET & & 31 & 80 & 64 \\
\hline
\end{tabular}

* Continuous current at $25^{\circ} \mathrm{C}$

** Typical ON resistance at $25^{\circ} \mathrm{C}$.

at $V_{\mathrm{DS}}=40 \mathrm{~V}$ versus frequency for devices A-D. Device A $(\mathrm{GaN})$ exhibits the lowest $R_{\mathrm{S}}$, which corresponds to the lowest $E_{\text {DIss. }}$ The method predicts the largest $C_{\text {oss }}$ losses for device D (SiC). To verify the prediction, we submitted devices $\mathrm{C}$ and $\mathrm{D}$ (with the same package) to a $75-\mathrm{V}$ peak-to-peak sine-wave at three different frequencies (1-MHz, 2-MHz, and $3-\mathrm{MHz})$ showing significantly higher losses in device $\mathrm{D}$, as predicted by the method (Figs. 3f and 3g).
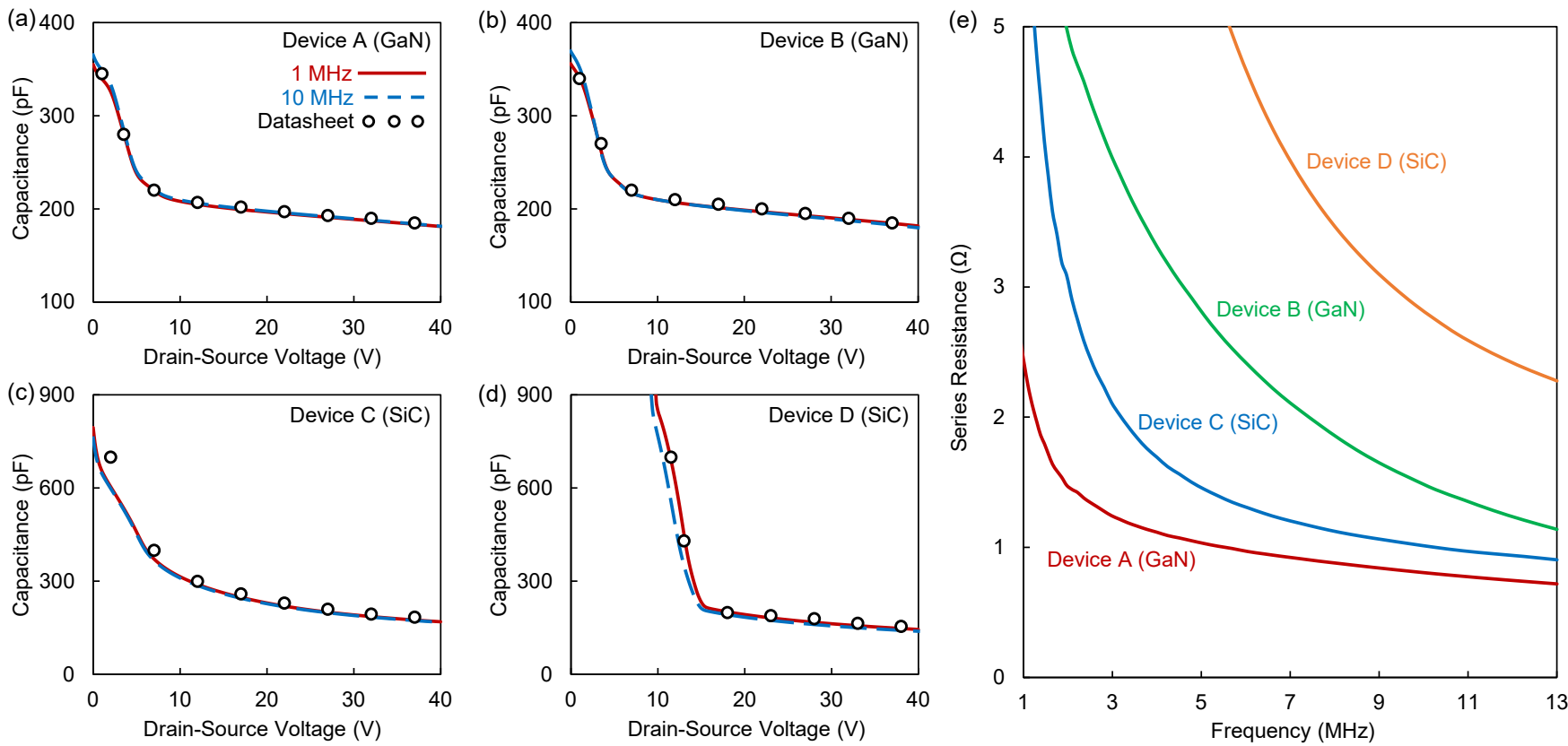

(f)

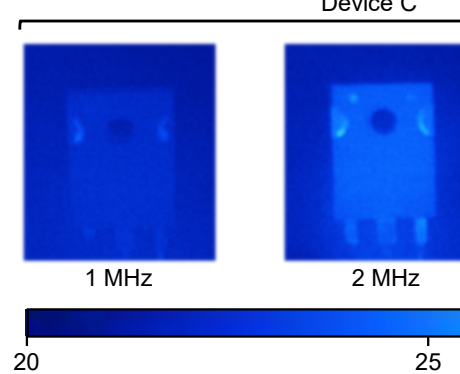

(g)

Device D

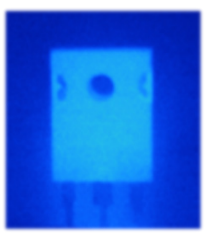

$3 \mathrm{MHz}$

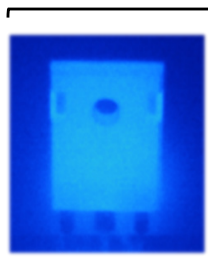

$1 \mathrm{MHz}$

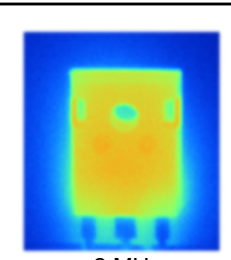

$2 \mathrm{MHz}$

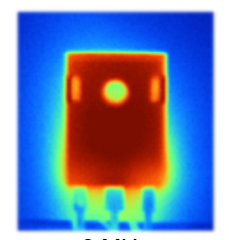

$3 \mathrm{MHz}$ $40^{\circ} \mathrm{C}$

Fig. 3. Measured $C_{\mathrm{OSS}}$ versus drain-source voltage at $1 \mathrm{MHz}$ (solid red line) and $10 \mathrm{MHz}$ (dashed blue line) as well as data reported in datasheet (discrete points) for (a) device A $(\mathrm{GaN})$, (b) device B $(\mathrm{GaN})$, (c) device C (SiC), and (d) device D (SiC), all 600/650-V rated with $\sim 30-\mathrm{A} \mathrm{current}$ rating (Table I). The figures show consistency of $C_{\mathrm{OSS}}$ over frequency as the valid assumption of the proposed method. (e) Series resistance $\left(R_{\mathrm{S}}\right)$ of the four devices versus frequency. The method simply predicts lowest and highest losses for devices A and D, respectively. Thermographs of (f) device $\mathrm{C}$ and (g) device D (gate and source shorted), both with the same package, submitted to $75-\mathrm{V}$ peak-to-peak sinusoidal waveform (charging and discharging $C_{\mathrm{OSS}}$ ) at three different frequencies $1 \mathrm{MHz}, 2 \mathrm{MHz}$, and $3 \mathrm{MHz}$. Device D shows considerably higher losses, as predicted by the proposed method.

(C) 2020 IEEE. Personal use of this material is permitted. Permission from IEEE must be obtained for all other uses, in any current or future media, including reprinting/republishing this material for advertising or promotional purposes, creating new collective works, for resale or redistribution to servers or lists, or reuse of any copyrighted component of this work in other works. 


\section{CONCLUSION}

We proposed a new $C_{\text {OSs }}$ model and measurement technique that enables selection of devices with lowest $C_{\text {OSS- }}$ losses among different WBG transistors, just by performing one small-signal measurement: $R_{\mathrm{S}}$-versus-frequency. It is also possible to use the measured $R_{\mathrm{S}}$ together with $C_{\mathrm{OSS}}^{\text {eff }}$ (which can be extracted from datasheet) to estimate the amount of $C_{\text {OsS }}$ losses. The generality and robustness of this method enables it to quantify $C_{\text {Oss }}$ losses of WBG transistors, which is a crucial source of losses in soft-switched power converters, and to select devices with the best performance.

\section{REFERENCES}

[1] T. P. Chow and R. Tyagi, "Wide bandgap compound semiconductors for superior high-voltage unipolar power devices," IEEE Trans. Electron. Devices, vol. 41, no. 8, pp. 1481-1483, Aug. 1994.

[2] S. Ji, D. Reusch, and F. C. Lee, "High-frequency high power density 3D integrated gallium-nitride-based point of load module design," IEEE Trans. Power Electron., vol. 28, no. 9, pp. 4216-4226, Sep. 2013.

[3] W. Chen, K. Wong, and K. J. Chen, "Single-chip boost converter using monolithically integrated $\mathrm{AlGaN} / \mathrm{GaN}$ lateral field-effect rectifier and normally off HEMT," IEEE Electron. Device Lett., vol. 30, no. 5, pp. 430-432, May 2009.

[4] G. Zulauf, S. Park, W. Liang, K. Surakitbovorn, and J. Rivas-Davila, "Coss losses in $600 \mathrm{~V}$ GaN power semiconductors in soft-switched, highand very-high-frequency power converters," IEEE Trans. Power Electron., vol. 33, no. 12, pp. 10748-10763, Dec. 2018.

[5] M. Guacci, et al. "On the origin of the $\mathrm{C}_{\text {oss }}$-losses in soft switching GaNon-Si power HEMTs," IEEE J. Emerg. Sel. Topics Power Electron., vol. 7, no. 2, pp. 679-694, Jun. 2019.

[6] G. Zulauf, Z. Tong, J. D. Plummer, and J. M. Rivas-Davila, "Active power device selection in high- and very-high-frequency power converters," IEEE Trans. Power Electron., vol. 34, no. 7, pp. 68186833, Jul. 2019

[7] M. Samizadeh Nikoo, A. Jafari, N. Perera, and E. Matioli, "Measurement of largesignal $C_{\mathrm{Oss}}$ and $C_{\mathrm{OSs}}$ losses of transistors based on nonlinear resonance," IEEE Trans. Power Electron., vol. 35, no. 3, pp. 2242-2246, Mar. 2020.

[8] M. Samizadeh Nikoo, A. Jafari, N. Perera, and E. Matioli, "New Insights on Output Capacitance Losses in Wide-Band-Gap Transistors," IEEE Trans. Power Electron., vol. 35, no. 7, pp. 6663-6667, Jul. 2020.

[9] K. Surakitbovorn and J. Rivas-Davila, "Evaluation of GaN transistor losses at MHz frequencies in soft switching converters," in Proc. IEEE 18th Workshop Control Modelling Power Electron. (COMPEL), Stanford, CA, USA, Jul. 2017.

[10] J. Fedison and M. Harrison, " $C_{\mathrm{O} \text { ss }}$ hysteresis in advanced superjunction MOSFETs," in Proc. Appl. Power Electron. Conf. Expo., 2016, pp. 247252.

[11] J. Fedison, M. Fornage, M. Harrison, and D. Zimmanck, " $C_{\text {oss }}$ related energy loss in power MOSFETs used in zero-voltage-switched applications," in Proc. 29th Annu. IEEE Appl. Power Electron. Conf. Expo., 2014, pp. 150-156. 